\title{
Mathematical Model of Evaluating the Teaching Skills
}

\author{
Congjun Rao, Benhe Yin \\ College of Mathematics and Physics, Huanggang Normal University, \\ Huanggang 438000, China \\ cjrao@163.com
}

Keywords: Teaching skills competition; Evaluation index system; Fuzzy comprehensive evaluation model

\begin{abstract}
With the development of curriculum reform, it is increasingly important to strengthen the training and evaluation of teaching skills for normal professional undergraduates. In this paper, the practical problem of evaluating the teaching skills competition for normal professional undergraduates is studied. Concretely, a new evaluation system of teaching skills competition is proposed, and a comprehensive evaluation model of teaching skills competition for normal professional undergraduates is established based on fuzzy mathematical theory, which provides a new way to evaluate the teaching level for normal professional undergraduates.
\end{abstract}

\section{Introduction}

With the reform of the education system, the competition of education jobs are becoming increasingly competitive and intense cruelty, the key factor of success is the teaching level. The development process of undergraduates from teaching posts, moved hire and promotion title everywhere are inseparable from this part of the lecture. In recent years, many teaching skills competition events have hold in many normal universities. How to choose the reasonable evaluation system and evaluation method for teaching skills competition is the preconditions to improve the teaching skills competition.

Taking the teaching process as the main line to construct a set of evaluation system for teaching skills competition can provide a scientific basis for the comprehensive and effective evaluation of normal professional undergraduates' teaching level. By the analysis of evaluation ratings for undergraduates' teaching skills, we can obtain the differences of teaching skills to various disciplines, which can play a guiding role for the training and evaluation of undergraduates' teaching skills. The effective implementation of evaluation system for undergraduates' teaching skills competition will help improve the quality of basic education teachers' training, so as to make an outstanding contribution by constructing high-quality basic education and innovative teachers. It also enhances the educational level in normal colleges and universities and effectively alleviates the employment pressure of undergraduates. It plays a positive role in promoting the teaching skills contest activities.

At present, the evaluation of teaching skills focused on two aspects: qualitative evaluation and quantitative evaluation. However, quantitative research also has its own shortcomings, i.e., some scholars have not taken into account the differences for various disciplines to undergraduates' teaching skills. For this shortcoming, Gao[] studied the problem of evaluating the classroom teaching skills by using the method of AHP. Moreover, there are many other shortcomings for the existing method of evaluating the undergraduates' teaching skills, i.e., the evaluation purposes lacks clarity, the evaluation lacks scientificity, the evaluation content lacks systematicness, the evaluation methods lack diversity, and the evaluation results lacks authority, which hindered the further promotion of undergraduates' teaching skills to some extent. Under this background, this paper proposes a new evaluation system of teaching skills competition, and establishes a comprehensive evaluation model of teaching skills competition for normal professional undergraduates based on fuzzy mathematical theory. It tries to provide new way to evaluate the teaching level for normal professional undergraduates. 


\section{Evaluation System of Teaching Skills Competition}

Analyzing the teaching skills classification at home and abroad, we can conclude that the first grade indexes can be divided into three aspects, i.e., teaching design skills before class, classroom teaching skills and teaching skills after class. According to the national issue " Training Program of Professional Skills for normal professional undergraduates", the classroom teaching skills includes introduction of teaching, explanation, questioning, presentation, writing on the blackboard, strengthen, feedback, end, change teaching skills and organizational skills. Based on above teaching skill categories, we establish a evaluation system of teaching skills competition for normal professional undergraduates which is listed in the following Table 1.

Table 1. Evaluation system of teaching skills competition for normal professional undergraduates

\begin{tabular}{ll}
\hline The primary evaluation attributes & \multicolumn{1}{c}{ The secondary evaluation attributes } \\
\hline$P_{1}$ Teaching design skills before class & $P_{11}$ Analysis of the teaching contents \\
& $P_{12}$ Analysis of the students \\
& $P_{13}$ Analysis of learning need \\
& $P_{14}$ Compiling of teaching goal \\
& $P_{15}$ Selection and design of teaching strategy \\
& $P_{16}$ Selection and usage of teaching media \\
& $P_{21}$ Introduction of teaching \\
& $P_{22}$ Explanation \\
& $P_{23}$ Teacher-student interaction \\
& $P_{24}$ Strengthen \\
& $P_{25}$ Demonstration \\
& $P_{26}$ Change control \\
& $P_{27}$ Evaluation and feedback \\
& $P_{28}$ Summary \\
$P_{2}$ Classroom teaching skills & $P_{31}$ Teacher's guidance after class \\
& $P_{32}$ Reconsider after teaching \\
\hline
\end{tabular}

\section{The Evaluation Model}

In this section, we will present a mathematical model to evaluate the teaching skills competition for normal professional undergraduates based on the method of fuzzy comprehensive evaluation. The basic steps are given as follows.

(1) Determine the evaluation grade set

$U=\left\{u_{1}, u_{2}, u_{3}, \ldots, u_{n}\right\}$;

Here we suppose that

$u_{1}=$ very good; $u_{2}=$ good; $u_{3}=$ common; $u_{4}=$ poor; $u_{5}=$ very poor;

(2) Determine the evaluation objects set

$V=\left\{v_{1}, v_{2}, v_{3}, \ldots, v_{m}\right\}$;

(3) Determine the evaluation attributes set. The primary evaluation attribute set is denoted as

$P=\left\{p_{1}, p_{2}, p_{3}\right\}$, 
and the secondary evaluation attribute set is denoted as

$$
\begin{aligned}
& p_{1}=\left\{p_{11}, p_{12}, \ldots, p_{16}\right\}, \\
& p_{2}=\left\{p_{21}, p_{22}, \ldots, p_{28}\right\}, \\
& p_{3}=\left\{p_{31}, p_{32}\right\}
\end{aligned}
$$

where the primary evaluation attributes and the secondary evaluation attributes are given in above Table 1.

(4) Determine the weights of evaluation attributes, i.e., use the quantitative way to reflect the important degree of various attributes in the evaluation.

The weight set of the primary evaluation attributes is denoted as

$$
W=\left\{W_{1}, W_{2}, W_{3}\right\} \text {. }
$$

and weight set of the secondary evaluation attribute set is denoted as

$$
\begin{aligned}
& w_{1}=\left\{w_{11}, w_{12}, \ldots, w_{16}\right\}, \\
& w_{2}=\left\{w_{21}, w_{22}, \ldots, w_{28}\right\}, \\
& w_{3}=\left\{w_{31}, w_{32}\right\} .
\end{aligned}
$$

(5) Determine the fuzzy relation matrix $R$ between the attributes and the evaluation grade set $U=\left\{u_{1}, u_{2}, u_{3}, u_{4}, u_{5}\right\}$ as follows.

$$
R=\left(\begin{array}{cccc}
r_{11} & r_{12} & \cdots & r_{1 m} \\
r_{21} & r_{22} & \cdots & r_{2 m} \\
\cdots & \cdots & \cdots & \cdots \\
r_{n 1} & r_{n 2} & \cdots & r_{n m}
\end{array}\right),
$$

where $0 \leq r_{i j} \leq 1$.

(6) Choose the composition operator to composite $A$ and $R$, then $B=\left(b_{1}, b_{2}, \cdots, b_{m}\right)$ is obtained, where

$$
\begin{gathered}
B=A O R=\left(a_{1}, a_{2}, \cdots, a_{n}\right) \mathrm{O}\left(\begin{array}{cccc}
r_{11} & r_{12} & \cdots & r_{1 m} \\
r_{21} & r_{22} & \cdots & r_{2 m} \\
\cdots & \cdots & \cdots & \cdots \\
r_{n 1} & r_{n 2} & \cdots & r_{n m}
\end{array}\right) \\
b_{j}=\left(a_{1} \bullet r_{1 j}\right)+\left(a_{2} \bullet r_{2 j}\right)+\cdots+\left(a_{n} \bullet r_{n j}\right), j=1,2, \cdots, m
\end{gathered}
$$

The commonly used fuzzy operators are as follows.

(i) $M(\wedge, \vee)$, i.e., substitute $\wedge$ into $\bullet_{*}$, substitute $\vee$ into ${ }_{*}^{+}$, where $\wedge$ is a minimizing operation, and $\vee$ is a maximum operation.

(ii) $M(\bullet, \vee)$, i.e., substitute the multiplication of real numbers into $\bullet_{*}$, and substitute $\vee$ into ${ }_{*}$;

(iii) $M(\wedge, \oplus)$, i.e., substitute $\wedge$ into $\bullet_{*}^{\bullet}$, and substitute $\oplus$ into ${ }_{*}^{+}$, where

$a \oplus b=\min (1, a+b)$;

(iv) $M(\bullet, \oplus)$ i.e., substitute the multiplication of real numbers into $\bullet_{*}$, and substitute $\oplus$ into ${ }_{*}$.

(7) Make a analysis for the results of fuzzy comprehensive evaluation for $B$.

Concretely, quantify the fuzzy evaluation grade evaluation, and calculate the priority of each evaluation object. Suppose that $u_{1}=$ very $\operatorname{good}=S_{1} ; u_{2}=\operatorname{good}=S_{2} ; u_{3}=\operatorname{common}=S_{3} ; u_{4}=$ poor $=S_{4}$; $u_{5}=$ very poor $=S_{5}$, then the priority degree of each object can be computed as follows.

$$
N_{k}=B_{k} S^{T}=\left(B_{k}^{1}, B_{k}^{2}, \cdots, B_{k}^{m}\right) \cdot\left(S_{1}, S_{2}, \cdots, S_{m}\right)^{T}
$$

Then we can rank the order according to the values of $N_{k}, k=1,2, \ldots, n$. The greater the value of $N_{k}$, the better the object $k$ is. 


\section{Conclusions}

In this paper, we study the problem of evaluating the teaching skills competition for normal professional undergraduates, and present a comprehensive evaluation model of teaching skills competition based on fuzzy mathematical theory. The evaluation model presented in this paper can be applied to the similar problems such as environmental pollution evaluation, wages level evaluation of enterprise staffs, prevention evaluation of drug abuse, school effectiveness evaluation, clinical evaluation and growth curve analysis.

\section{Acknowledgments}

This work is supported by the Natural Science Foundation of Hubei Province (No.2014CFC1096), and the 2014 Key Project of Hubei Provincial Department of Education (No.D20142903).

\section{References}

[1] V. Kucsera and M. Svinicki: The Journal of Faculty Development Vol. 24 (2010), p. 5-18.

[2] S.R. Hines: The Journal of Faculty Development Vol. 23 (2009), p. 5-19.

[3] J. Butcher and D. Stoncel: International Journal for Academic Development Vol. 17 (2012), p. 149-162.

[4] G. Gibbs: The International Journal for Academic Development Vol. 18 (2012), p. 4-14.

[5] Y. Xun: Shanghai Education and Research Vol. 5 (2004), p. 24-29.

[6] M. Yu: Classroom Teaching Skills and Countermeasures of Young University Teachers (Suzhou University thesis, Jiangsu, 2007).

[7] J.L. Shen and K.R. Wang: Journal of Beijing Normal University (Humanities and Social Science Edition) Vol. 34 (2000), p. 66-68.

[8] X.M. Zhong: Teacher Education Research Vol. 34 (2005), p. 31-35. 